\title{
Coronary artery stenosis regression by long-term standard dose statin therapy in middle-aged and elderly patients: Report and analysis of 31 cases
}

\author{
Zhilei Ren ${ }^{\mathrm{a}}$, Chongzhe Yang ${ }^{\mathrm{a}}$, Kaihua Huang ${ }^{\mathrm{a}}$, Feng Liu $^{\mathrm{a}^{*}}$ \\ ${ }^{a}$ National Key Clinical Department of Gerontology, Guangzhou First People's Hospital, School of Medicine, South China \\ University of Technology, Guangzhou, 510180, Guangdong, China.
}

\begin{abstract}
Background: To study the possibility and characteristics of long-term standard dose statin-induced stenosis regression in middle-aged and elderly patients with coronary heart disease (CHD).

Methods: The study patients were those with CHD admitted to our hospital from 2010 to 2019. All patients underwent computed tomography angiography (CTA) scanning and were diagnosed with a regression of stenosis.

Results: Thirty-one patients (24 males and 7 females) with an average age of $64.7 \pm 1.8$ years were reexamined by CTA at a mean of $18.6 \pm 1.9$ months after the initial examination. The left anterior descending (LAD) coronal artery stenoses were significantly regressed.

Conclusion: In middle-aged and elderly patients with CHD, a long-term and standard dose statin treatment may lead to the regression of coronary artery stenosis.

Keywords: Coronary atherosclerosis, statin, coronary CT angiography, middle-aged and elderly
\end{abstract}

\section{Introduction}

In the treatment of coronary heart disease (CHD), antiplatelet drugs, HMG-CoA reductase inhibitors (statins), angiotensin-converting enzyme inhibitors (ACEI), and beta receptor blockers $(\beta \mathrm{RB})$ can improve the prognosis. However, few studies have reported on the clinical effect of these drugs on coronary artery stenosis. The limited studies typically report the use of coronary angiography and intravascular ultrasound, whereas the use of noninvasive coronary computed tomography angiography (CTA) is reported less frequently. Compared with the gold standard of coronary angiography, the use of coronary CTA has increased by up to $96 \%$ in the diagnosis of CHD [1-6]. In 2013, our department Director, Feng Liu, treated a pa-

\footnotetext{
* Corresponding author: Feng Liu

Mailing address: National Key Clinical Department of Gerontology, Guangzhou First People's Hospital, School of Medicine, South China University of Technology, Guangzhou, 510180, Guangdong, China.

E-mail: eyliufeng@scut.edu.cn
}

Received: 07 September 2020 / Accepted: 22 September 2020 tient with CHD with pravastatin (40 mg, once nightly). After the patient's symptoms improved, he reexamined the patient with coronary CTA and found that more than $90 \%$ of the original stenosis had completely disappeared. He then had the idea of making further observations of the therapeutic effect of statins on various degrees of coronary stenosis.

Many outpatients and inpatients in the geriatrics department of our hospital have complaints of chest tightness and chest pain symptoms but have no indication of a need for emergency percutaneous coronary intervention (PCI). For the newly diagnosed patients, we should aim to recommend a coronary CTA examination. If diagnosed with coronary artery stenosis and CHD, they should accept the routine treatment recommended by the guidelines. Coronary CTA is recommended when the condition is stable for about one year. If coronary artery stenosis does not meet the diagnostic criteria of CHD, if other risk factors are present, and if the patient has no contraindication for the use of statins, we suggest the use of a conventional dose of statins and a repeat and reevaluation of the coronary CTA after about 1 year.

In the present study, a total of 31 patients who took a conventional dose of statins for $18.6 \pm 1.9$ months and completed two coronary CTA evaluations before and after coronary angiography met the requirements of reversing stenosis. 


\section{Clinical Data}

\section{General patient information}

The patients included 24 males and 7 females with an average age of $64.7 \pm 1.8$ years. nAccording to the CHD classification, 15 cases were stable angina pectoris, 12 cases were unstable angina pectoris, 1 case was occult CHD, 1 case was ischemic cardiomyopathy, and 2 cases were only coronary artery stenosis. Among the 31 patients, 21 had hypertension history and 13 had diabetes mellitus or impaired glucose tolerance. In all, 4 patients took oral pravastatin $(40 \mathrm{mg}), 8$ patients took rosuvastatin $(10 \mathrm{mg}), 12$ patients took atorvastatin $(20 \mathrm{mg}), 1$ case took atorvastatin $(10 \mathrm{mg}), 1$ case took simvastatin $(20 \mathrm{mg}), 1$ case took pivastatin $(2 \mathrm{mg})$, and 4 cases changed two or more statins before and after treatment. None of the patients took any lipid-lowering drugs other than statins during the treatment. The average interval from the first CTA examination to the reexamination was $18.6 \pm 1.9$ months.

\section{Follow-up of coronary CTA}

All 31 patients underwent coronary CTA with an Aquilion One 320 slice CT. At the same time, blood samples were taken to measure cholesterol and triglyceride (TG) levels. After reexamination, if the stenosis of one or more coronary branches was decreased from $>70 \%$ to less than $50 \%$, or if the stenosis was $50-70 \%$, or if the stenosis degree was reduced by more than $30 \%$ compared with the previous value, then stenosis was deemed to be reversed [78]. A paired t-test was used to compare the values before and after the reexamination, with $\mathrm{P}<0.05$ indicating a significant statistical difference. The cardiac function (left ventricular ejection fraction [LVEF], left ventricle at enddiastole [LVDd], and cardiac output [CO]) had no obvious deterioration or improvement. The degree of stenosis of the main coronary branches was significantly improved. The degree of stenosis of the left anterior descending branch was significantly reduced from $48.7 \pm 5.7 \%$ to 33.4 $\pm 4.4 \%(\mathrm{P}=0.04)$. The average degree of stenosis of the left circumflex artery decreased from $31.8 \pm 6.2 \%$ to 23.8 $\pm 5.5 \%$. No significant difference was observed in the left circumflex artery $(\mathrm{P}=0.34)$. The mean degree of stenosis of the right coronary artery decreased from $37.2 \pm 5.9 \%$ to $24.5 \pm 4.4 \%$, and no significant difference was noted in the right coronary artery $(\mathrm{P}=0.09)$.

\section{Follow-up of blood lipid efficacy}

The comparison of blood lipid levels determined at the same time as the CTA. Comparison of the changes in blood lipids with a paired t-test indicated a statistically significant difference $(\mathrm{P}<0.05)$. The levels in low density lipoprotein (LDL-C, $2.48 \pm 0.21$ vs. $2.28 \pm 0.15 \mathrm{mmol} / \mathrm{L}$ ), high-density lipoprotein (HDL-C, $1.08 \pm 0.05$ vs. $1.08 \pm$ $0.06 \mathrm{mmol} / \mathrm{L}$ ), total cholesterol (TC, $6.70 \pm 3.59$ vs. 3.33 $\pm 0.34 \mathrm{mmol} / \mathrm{L}$ ) and triglyceride (TG, $3.48 \pm 0.35 \mathrm{vs} .3 .23$ $\pm 0.30 \mathrm{mmol} / \mathrm{L})$ showed no significant changes compared with those at the initial diagnosis $(\mathrm{P}>0.05)$.

\section{Discussion}

CHD includes a series of symptoms of acute myocardial ischemia, chronic ischemia, or paroxysmal ischemia caused by coronary artery stenosis or spasm or to plaque rupture due to coronary atherosclerosis. The idea behind curing coronary heart disease should be to reduce or eliminate coronary stenosis and to control coronary atherosclerosis. Percutaneous coronary stent implantation, rotational milling of the stenosis site, and coronary artery bypass grafting are commonly used external intervention methods. The basis of external interventions should be basic drug treatment, and statins are one of the usual drugs administered to improve the prognosis.

Atherosclerosis is mainly caused by dyslipidemia and vasculitis. Statins can effectively regulate blood lipids, inhibit vascular inflammation, and improve endothelial function, thereby stabilizing or even reversing coronary atherosclerosis [9]. A number of studies have shown that intensive statin lipid-lowering therapy can delay or even reverse atherosclerosis [10-13]. In the East Asian population, conventional-dose statins may also reverse plaque [14-15]. However, few reports have examined whether the use of conventional statins can reduce stenosis.

In 2013, we found a case of coronary artery stenosis reversal by coronary CTA after one year of a conventional dose of pravastatin. We have since summarized 31 cases of elderly patients with coronary stenosis reversal confirmed by CTA review in our department since 2010, and we have analyzed their characteristics. In view of the fact that most of these patients admitted to our department are middle-aged or elderly patients, the risk of adverse reactions should be considered regarding the treatment with statins. For this reason, the treatment dose is typically the conventional dose specified in the guidelines. The average age of these 31 patients was $64.7 \pm 1.8$ years old, and the average application time was $18.6 \pm 1.9$ months. After long-term regular doses of statins, all patients showed results indicating a reversal of coronary artery stenosis.

Low density lipoprotein cholesterol (LDL-C) is an important promoter of atherosclerosis. A number of largescale, multicenter, randomized double-blind trials have confirmed that statins, as the core drugs for primary and secondary prevention of atherosclerotic cardiovascular disease (ASCVD), can reduce the incidence of adverse cardiovascular events. A meta-analysis showed that the clinical cardiovascular benefits of statins depended on the decrease in LDL-C and had no relationship with the type of statins [16]. Our results showed that the low-density lipoprotein level of the selected patients did not significantly decrease during the observation period after longterm oral statin use. This difference may reflect the fact that the selected patients had been treated with statins for a long time at the beginning of the observation, so their blood lipids had stabilized at a certain level. In addition, the serum levels of HDL, TC, and TG did not change significantly during the observation period. On the one hand, 
long-term use of statins plays an important role in the secondary prevention of coronary heart disease. On the other hand, the patient may benefit from the lipid-lowering effects of statins, such as their anti-inflammatory effect, improvement of endothelial function, etc. Patients with CHD need to adhere to their statins regimen to benefit from the secondary prevention roles of these drugs.

In terms of diagnosis and efficacy, a variety of imaging detection methods can directly assess the severity and progression of coronary artery stenosis plaque. Compared with coronary CTA, coronary angiography is still the "gold standard" for the diagnosis of coronary heart disease. Although intravascular ultrasound (IVUS) and optical coherence tomography (OCT) have their own benefits [17], the advantages of coronary CTA lie in its noninvasive nature, its relatively lower cost, and its faster speed, which make it more conducive for screening and follow-up of the CHD population. In patients with acute chest pain, a CT examination can reveal whether a cerebral embolism is present along with a pulmonary embolism. The coronary calcification score and fractional flow reserve (FFR) data of CTA are also closely related to the patient's prognosis. At present, ischemia evaluation is expected to further enhance the role of CTA as an important examination method in patients with CHD. This is the reason why we chose coronary CTA as the method to evaluate the reversal of stenosis.

The existing large-scale clinical trials rarely include elderly patients; therefore, the clinical efficacy and safety of statins need more evidence-based medicine. The longterm use of statins in elderly patients, in particular, needs to be assessed for possible risks, which can include transient or persistent liver damage, myalgia (and even rhabdomyolysis), a slightly increased risk of diabetes, and mild to moderate peripheral and central nervous system symptoms. However, our treatment of 31 elderly patients showed that if we use conventional doses of statins and pay close attention to the level of liver transaminase and creatine kinase, to the blood glucose level, and to possible symptoms of these patients, most elderly patients can tolerate any adverse events that may occur by changing the type of statins, reducing the dosage, or adjusting other oral drugs. The benefits will far outweigh the risks.

The 31 patients with coronary artery stenosis including CHD were treated with a conventional dose of statins. Through long-term follow-up, in addition to the obvious control of symptoms and stable clinical condition, the improvement in coronary CTA provides us with a first-line hope of curing chronic heart disease through drugs.

\section{Declarations}

Acknowledgments: We thank Dr. Wei Ma and Dr. Luqian Zhao for the professional suggestions.

Authors' contributions: Zhilei Ren and Chongzhe Yang contributed equally to this article. Made substantial contri- butions to conception and design of the study: Feng Liu; Performed data acquisition, as well as provided administrative, technical, and material support: Zhilei Ren, Kaihua Huang. Performed data analysis and interpretation, Performed article writing: Zhilei Ren, Chongzhe Yang.

Financial support and sponsorship: This work was supported by Guangzhou Planed Project of Science and Technology (201704020105) to Feng Liu.

Conflicts of interest: All authors declared that there are no conflicts of interest.

Ethical approval and consent to participate: This is a retrospective study. We're just using the data that patients already have without adding interventions. No ethics involved.

\section{References}

1. Lee S I, Miller J C, Abbara S, et al. Coronary CT angiography. Journal of the American College of Radiology, 2006, 3(7): 560-564.

2. Arbab-Zadeh A, Carli M F D, Cerci R, et al. Accuracy of computed tomographic angiography and single-photon emission computed tomography-acquired myocardial perfusion imaging for the diagnosis of coronary artery disease. Circulation: Cardiovascular Imaging, 2015, 8(10): e003533.

3. Machida H, Tanaka I, Fukui R, et al. Current and novel imaging techniques in coronary CT. Radiographics, 2015, 35(4): 991-1010.

4. Alfakih K, Plein S. PROMISE: where now for CT coronary angiography. 2015.

5. Menke J, Kowalski J. Diagnostic accuracy and utility of coronary CT angiography with consideration of unevaluable results: a systematic review and multivariate Bayesian random-effects meta-analysis with intention to diagnose. European radiology, 2016, 26(2): 451-458.

6. Ko B S, Wong D T L, Nørgaard B L, et al. Diagnostic performance of transluminal attenuation gradient and noninvasive fractional flow reserve derived from 320-Detector Row CT angiography to diagnose hemodynamically significant coronary stenosis: an NXT substudy. Radiology, 2016, 279(1): 75-83.

7. Hangartner J R, Charleston A J, Davies M J, et al. Morphological characteristics of clinically significant coronary artery stenosis in stable angina. Heart, 1986, 56(6): 501508.

8. Park S J, Park S W, Hong M K, et al. Long-term (threeyear) outcomes after stenting of unprotected left main coronary artery stenosis in patients with normal left ventricular function. The American journal of cardiology, 2003, 91(1): 12-16.

9. Liao $\mathrm{YH}$, Cheng X, Huang $\mathrm{K}$, et al. Expert consensus on statin-induced plaque regression in ASCVD. J Clin Cardiol (China). 2015. 31(1): 1-5.

10. Nissen S E. REVERSAL Investigators: Effect of intensive compared with moderate lipid-lowering therapy on 
progression of coronary atherosclerosis: a randomized controlled trial. JAMA, 2004, 291: 1071-1080.

11. Nissen S E, Nicholls S J, Sipahi I, et al. Effect of very highintensity statin therapy on regression of coronary atherosclerosis: the ASTEROID trial. Jama, 2006, 295(13): 1556-1565.

12. Räber L, Taniwaki M, Zaugg S, et al. Effect of high-intensity statin therapy on atherosclerosis in non-infarctrelated coronary arteries (IBIS-4): a serial intravascular ultrasonography study. European heart journal, 2015, 36(8): 490-500.

13. Nicholls S J, Ballantyne C M, Barter P J, et al. Effect of two intensive statin regimens on progression of coronary disease. New England Journal of Medicine, 2011, 365(22): 2078-2087.

14. Takayama T, Hiro T, Yamagishi M, et al. Rationale and Design for a Study Using Intravascular Ultrasound to Evalu- ate Effects of Rosuvastatin on Coronary Artery Atheroma in Japanese Subjects. Circulation Journal, 2007, 71(2): 271-275.

15. Lee C W, Kang S J, Ahn J M, et al. Comparison of effects of atorvastatin $(20 \mathrm{mg})$ versus rosuvastatin $(10 \mathrm{mg})$ therapy on mild coronary atherosclerotic plaques (from the ARTMAP trial). The American journal of cardiology, 2012, 109(12): 1700-1704.

16. Trialists $C \mathrm{~T}$. Efficacy and safety of more intensive lowering of LDL cholesterol: a meta-analysis of data from 170 000 participants in 26 randomised trials. The Lancet, 2010, 376(9753): 1670-1681.

17. Pasterkamp G, Den Ruijter H M, Libby P. Temporal shifts in clinical presentation and underlying mechanisms of atherosclerotic disease. Nature Reviews Cardiology, 2017, 14(1): 21-29.

Cite this article as: Ren Z, Yang C, Huang K, et al. Coronary artery stenosis regression by long-term standard dose statin therapy in middle-aged and elderly patients: Report and analysis of 31 cases[J]. Aging Pathobiology and Therapeutics, 2020, 2(3): 162-165. 